\title{
Femtosecond Written Silica Waveguides for High Extinction Polarization Filtering
}

\author{
Timothy Lee ${ }^{1 *}$, Qi Sun ${ }^{1}$, Rand Ismaeel ${ }^{1}$, Marta Castro-Lopez ${ }^{2}$, Cao Bocheng ${ }^{3}$, \\ Martynas Beresna ${ }^{1}$, Gilberto Brambilla ${ }^{1}$ \\ ${ }^{1}$ Optoelectronics Research Centre, University of Southampton, Southampton, SO17 1BJ, UK \\ ${ }^{2}$ Huawei Technologies Duesseldorf GmbH, Riesstr. 25, C3, 80992, Munich, Germany \\ ${ }^{3}$ Huawei Technologies Japan KK, Yokohama, Japan \\ *tl305@soton.ac.uk
}

\begin{abstract}
Silica waveguides with polarization-selective transmission were written by femtosecond laser inscription using linear and circular polarization. By tailoring writing parameters, polarization-dependent losses from 0.5 to $24 \mathrm{~dB}$ at $1310 \mathrm{~nm}$ were achieved.

OCIS codes: (230.7370) Waveguides; (130.5440) Polarization-selective devices; (140.3390) Laser materials processing
\end{abstract}

The last decade has seen growing research and industrial interest towards femtosecond writing, which relies on focusing high power pulses into a substrate where nonlinear absorption effects localized at the focal volume result in a permanent refractive index modification [1]. Thus, complex 3D structures can be rapidly designed, implemented and tested, such as waveguides, gratings and multiplexers [2-4]. To expand the scope, additional functionality for signal processing or filtering is important.

We report here on the fabrication and demonstration of waveguides with polarization dependent transmission, i.e. which transmit one polarization well but strongly attenuate the other. The geometry is a straightforward stepindex buried waveguide, which allows easy and compact incorporation with integrated optics for applications necessitating polarization filtering, such as sensing or polarization division demultiplexing signal cleaning.

Waveguides were inscribed using $1030 \mathrm{~nm}$ wavelength $200 \mathrm{fs}$ pulses from a Pharos 05-200-PP laser (Light Conversion Ltd., Lithuania) focused by an objective (Leitz Wetzlar $\times 25$ 0.4NA PLAN), as shown in Fig. 1 . The first half-waveplate and polarizing beamsplitter cube control power, while a second half-waveplate adjusts polarization angle. Both linear and circular polarization writing beams were investigated; the latter by inserting a quarterwaveplate before the objective. The multiscan method [2] was used to create $10 \mu \mathrm{m}$ wide waveguides by scanning 50 lines spaced $200 \mathrm{~nm}$ apart. All waveguides were $10 \mathrm{~mm}$ long and inscribed $500 \mu \mathrm{m}$ beneath the surface, in fused silica substrates (Spectrosil 2000, Heraeus). After writing, both ends were lapped to expose the facets and polished.

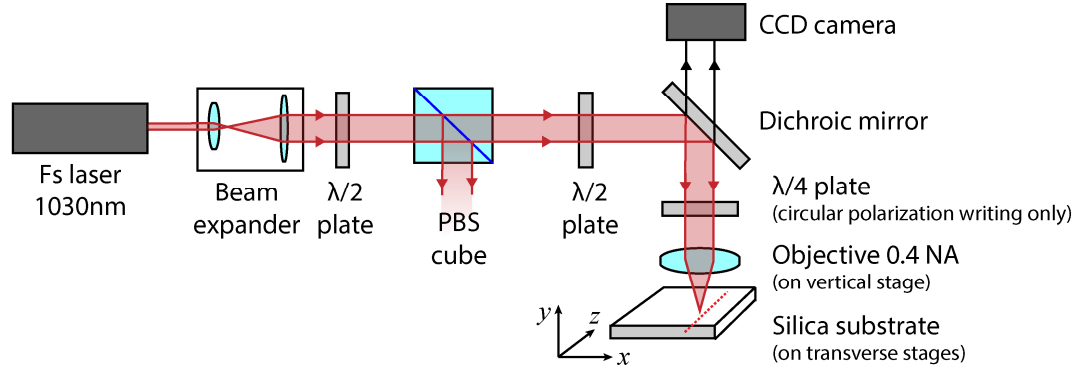

Fig. 1. Femtosecond writing set up for fabricating waveguides. The $x$ and $y$ axes are transverse to the waveguide direction $z$, and the writing beam is aligned with the $y$ axis.

The objective and sample are both mounted on precision translation stages (Aerotech Ltd.) and the laser pulse firing is synchronized to the sample position. Thus, the pulse density $D$, i.e. number of pulses per $\mathrm{mm}$ along the waveguide, can be conveniently adjusted independently of writing velocity $v$. In addition, the pulse burst $k_{B}$ can be set so the laser fires multiple times at each point. For example, $D=10^{4} \mathrm{~mm}^{-1}$ with $k_{B}=3$ implies that every $100 \mathrm{~nm}$, the laser will fire 3 pulses (in the same spot). The product $D \cdot k_{B} \cdot v$ cannot exceed the laser maximum repetition rate of $200 \mathrm{kHz}$. Note that this relatively low pulse frequency means no significant cumulative heating is observed [5].

To characterize the waveguides, CW light from a $1310 \mathrm{~nm}$ laser diode was launched into a linear polarizer and half-waveplate to control polarization, before being butt-coupled via polarization maintaining (PM) fiber into the waveguide. The output was focused by a $0.6 \mathrm{NA}$ objective onto either a CCD or power meter, for mode field diameter (MFD) or loss measurements, respectively. 
All waveguides were written with a $10 \times 10 \mu \mathrm{m}$ square cross-section core as shown in Fig. 2(a), and in each case, the $y$ polarization was found to transmit better than the $x$ polarization which experienced scattering. Examples of the transmitted $y$ polarized mode intensity profile are given in Fig. 2(b) and (c).

(a)

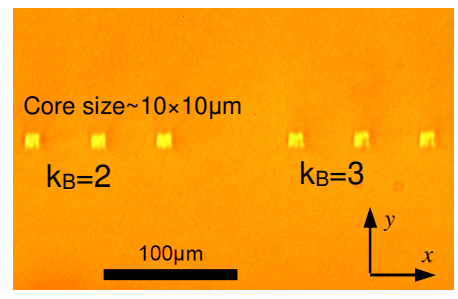

(b)

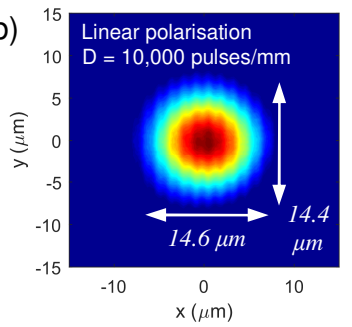

$\begin{array}{lll}\text { (C) }{ }_{10}^{15} & \text { Circular polarisation } \\ & \mathrm{D}=200,000 \text { pulses } / \mathrm{mm}\end{array}$

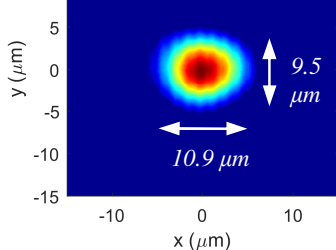

Fig. 2. (a) Microscope image of waveguide cross sections, written by linear polarisation for $k_{B}=2$ and 3. (b) $y$-polarised mode intensity profile for $D=1 \times 10^{4}$ pulses $/ \mathrm{mm}$ written using linear polarisation and (c) $D=2 \times 10^{5} \mathrm{pulses} / \mathrm{mm}$ using circular polarisation. The $1 / \mathrm{e}^{2}$ MFD is labelled.

For a writing beam linearly polarized perpendicular to waveguide direction, Fig. 3(a) shows that as pulse density increases from 5000 to 15000 pulses $/ \mathrm{mm}$ (while keeping velocity constant at $5 \mathrm{~mm} / \mathrm{s}$ and pulse energy at $300 \mathrm{~nJ}$ ), the $y$ polarized mode loss remains low in the range $\alpha_{y}=1.2-1.5 \mathrm{~dB}$, whereas the $x$ polarization loss increases steeply from $\alpha_{x}=1.6$ to $13.0 \mathrm{~dB}$ due to increased scattering. The scattering is believed to arise from the presence of anisotropic nanostructures $[1,6]$ formed under higher pulse density regimes, which can be adjusted to engineer a desired extinction ratio between the transmitted and scattered polarization. Thus, the polarization dependent loss (PDL) increases from only 0.5 up to $11.5 \mathrm{~dB}$. Likewise, increasing $k_{B}$ from 1 to 4 causes a similar effect shown in Fig. 3(b) with PDL reaching $12.7 \mathrm{~dB}$, while $\alpha_{y}$ is kept small in the range $1.3-2.1 \mathrm{~dB}$.

(a)

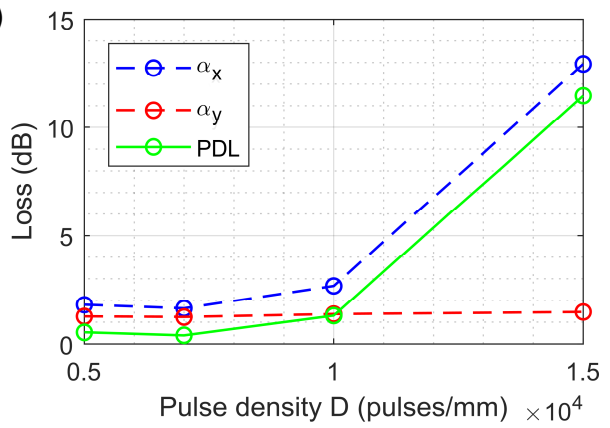

(b)

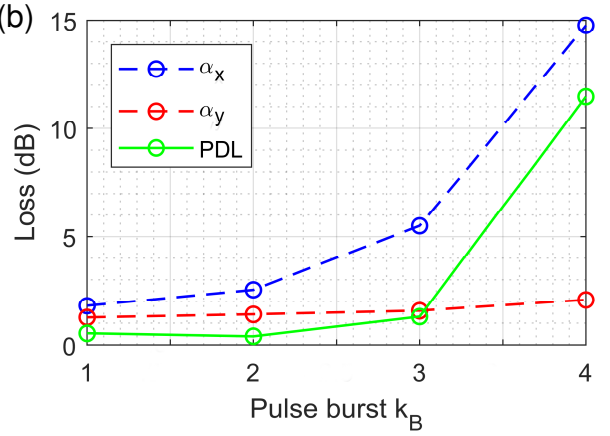

Fig. 3. Waveguide insertion loss measured with $x$ and $y$ polarizations and polarization-dependent loss against (a) writing pulse density, with $k_{B}=1$ and (b) pulse burst, with $D=5000$ pulses $/ \mathrm{mm}$. Written with perpendicular linear polarisation at velocity of $5 \mathrm{~mm} / \mathrm{s}$. Note: insertion loss values include fibre-waveguide coupling loss and Fresnel reflection loss.

Increasing either $D$ or $k_{B}$ beyond these ranges results in damage and high loss for both polarizations. However, writing with circular polarization allows much higher pulse densities to be tolerated. For $D=200,000$ pulses $/ \mathrm{mm}$ $\left(v=1 \mathrm{~mm} / \mathrm{s}, k_{B}=1\right.$ and pulse energy at $300 \mathrm{~nJ}$ ), the waveguide losses are $\alpha_{y}=2.5 \mathrm{~dB}$ and $\alpha_{x}=26.8 \mathrm{~dB}$, thus achieving a high PDL of $24.3 \mathrm{~dB}$. These waveguides are well suited for polarization filtering requiring high extinction.

In conclusion, waveguides which selectively transmit the $y$ polarization and filter out the $x$ polarization, with an extinction up to $24 \mathrm{~dB}$ at $1310 \mathrm{~nm}$, were fabricated by femtosecond writing at $1030 \mathrm{~nm}$. Both linear and circular writing polarizations were investigated, with circular polarization offering higher extinction. The mechanism relies on a polarization dependent scattering effect enhanced by writing at higher pulse densities of $10^{4}-10^{5}$ pulses $/ \mathrm{mm}$.

\section{References}

[1] M. Beresna et al., "Ultrafast laser direct writing and nanostructuring in transparent materials," Adv. Opt. Photon. 6, 293-339 (2014).

[2] Y. Nasu et al., "Low-loss waveguides written with a femtosecond laser for flexible interconnection in a planar light-wave circuit," Opt. Lett. 30, 723-725 (2005).

[3] A. Donko et. al., "Point-by-point femtosecond laser micro-processing of independent core-specific fiber Bragg gratings in a multi-core fiber," Opt. Express 26, 2039-2044 (2018).

[4] V. A. Amorim et al., "Monolithic Add-Drop Multiplexers in Fused Silica Fabricated by Femtosecond Laser Direct Writing," J. Lightwave Technol. 35, 3615-3621 (2017).

[5] S. M. Eaton et al., "Heat accumulation effects in femtosecond laser-written waveguides with variable repetition rate," Opt. Express 13, 4708$4716(2005)$.

[6] G. Cheng et al., "Ultrafast laser photoinscription of polarization sensitive devices in bulk silica glass," Opt. Express 17, 9515-9525 (2009). 